\title{
INVITED DISCOURSES
}




\title{
A NEW LOOK AT THE SUN
}

\author{
J. P. WILD
}

CSIRO, Division of Radiophysics, Epping, N.S.W., Australia

I have the feeling that to most astronomers the Sun is rather a nuisance. The reasons are quite complex. In the first place the Sun at once halves the astronomer's observing time from 24 to 12 hours, and then during most of the rest of the time it continues its perversity by illuminating the Moon. Furthermore I have met numerous astronomers who regard solar astronomy to be now, as always before, in a permanent state of decline - rather like Viennese music or English cricket. Nevertheless those who study the Sun and its planetary system occasionally make significant contributions. There were, for instance, Galileo and Newton who gave us mechanics and gravitation; Fraunhofer who gave us atomic spectra; Eddington and Bethe who pointed the way to nuclear energy; and Alfven who gave us magneto-hydrodynamics. Perhaps the point to be recognized is that the Sun has more immediately to offer to physics rather than to astronomy. That is why it is quite rare that a solar man finds himself with a large captive audience of mainline astronomers: and so the responsibility weighs heavily on my shoulders tonight.

My theme will in fact be a very simple and clearly defined one. It is to describe the attempts of my colleagues and me to produce pictures - moving pictures - of the Sun in the metre wavelength part of the radio spectrum and to tell you about the results which have emerged from these observations. The instrument in question, known as a radioheliograph, is located 300 miles northwest of Sydney at Culgoora observatory which many of you will be visiting around this time. I would like you to consider my personal role in all this as that of a spokesman speaking on behalf of a research group. I should acknowledge in particular the instrumental work of Kevin Sheridan (without whom the thing would never have worked), Maston Beard, Keith McAlister and Warren Payten; and the research work of Steve Smerd, Norman Labrum, Donald McLean, Anthony Riddle, Ron Stewart; and also invaluable contributions by people from other countries - from Dr Morimoto, and Dr Kai of Tokyo Astronomical Observatory and Dr George Dulk from the University of Colorado. Those are a few names, but the list could be made actually much longer, of people who have played quite essential parts in this work.

\section{The Culgoora Radioheliograph}

The first germs of the idea of producing a radioheliograph came, as far as I was concerned, in the late 1950's when, after spending a decade or so studying the radio waves from the Sun by indirect and devious ways, the thought kept occurring:wouldn't it be fun if we could actually look at or take pictures of the Sun, with a kind of radio movie camera. And to understand how to specify this radio camera 
we have to look first at what had already been established, what information was available at that time about the general properties of the radio emission.

As you all know it had been established that from time to time - significantly enough at the time of solar flares - the 'quiet' level of solar radio emission would suddenly increase by a factor of hundreds or even thousands; the intensity of such an outburst was found to be equivalent to an object of the size of the Sun radiating as a black body at hundreds of millions of degrees. One was clearly dealing with nonthermal processes and a lot of work and soul-searching went on during the forties and fifties to explore the mechanisms and processes responsible. The outcome is illustrated in Figure 1 which summarizes ten or more years of rather tough horsetrading between the observationalists and the theorists. It was concluded that there are three types of emission, all produced by electrons of different energy. First there are the thermal electrons in the solar atmosphere which produce bremsstrahlung of a comparatively very feeble intensity, merely that of a black body of the order of a million degrees. Then we have the supra-thermal electrons in the energy range

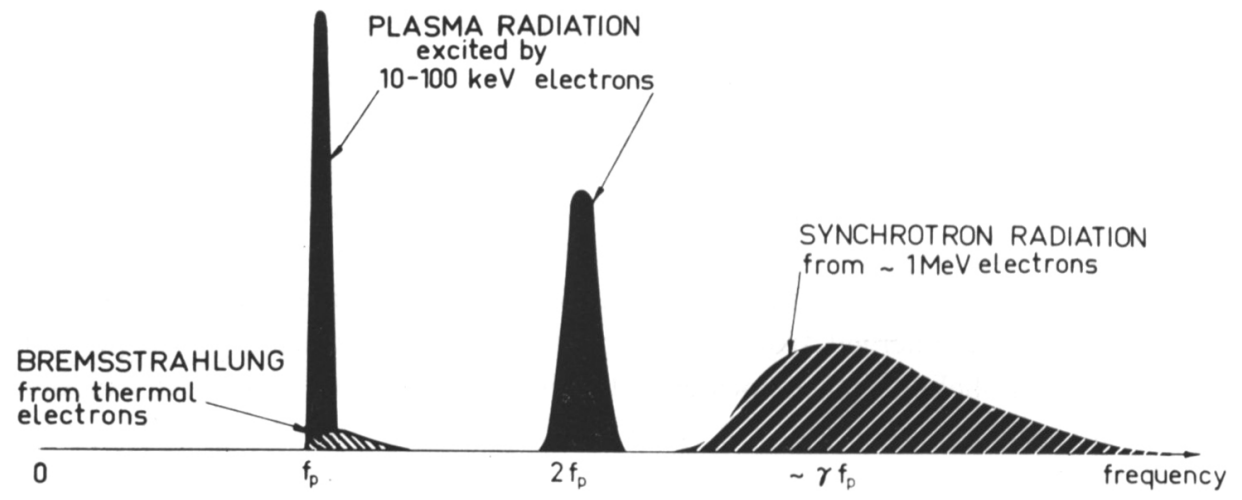

Fig. 1. Idealized spectrum of the three types of radiation believed to be responsible for solar radio emission.

10 to $100 \mathrm{keV}$ which, by a series of processes which the theorists argue about at very great length, set the solar atmosphere into plasma oscillations which produce radiation at two sharp frequency resonances, one at the plasma frequency $f_{p}$, and another at the second harmonic, $2 f_{p}$.

Such plasma radiation provides us with an inherently powerful tool for exploring the solar atmosphere because different frequencies are emitted from regions of different electron-density, $N$, ie. from different levels - according to the well - known formula

$$
f_{p}=e \sqrt{\frac{N}{\pi m}}
$$

where $e$ and $m$ denote electronic charge and mass. Typical isodensity contours of the solar corona are shown in Figure 2, plotted in terms of $f_{p}$ rather than $N$. Finally we 
have the relativistic electrons with energies in the $\mathrm{MeV}$ range, and these generate synchrotron radiation. To those of you who study synchrotron radiation emitted by cosmic radio sources the spectrum shown in Figure 1 may look unfamiliar. The reason is that in the solar environment the low frequency cutoff is determined by the refractive index of the medium (corona) which suppresses the low frequencies; all that remains is a frequency tail situated around a frequency a few times the plasma frequency.

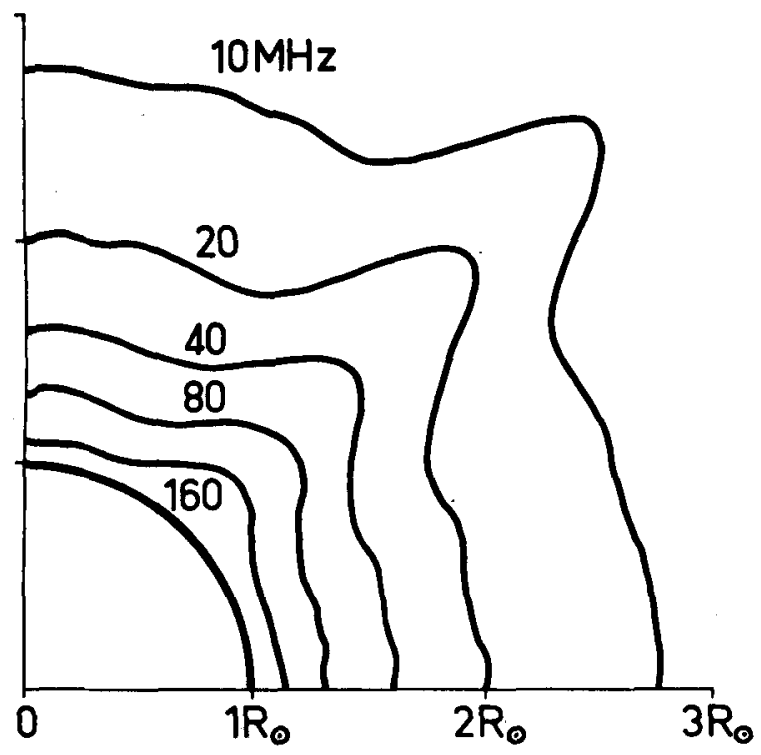

Fig. 2. Typical electron-density contours of the solar corona designated in terms of their plasma frequency in accordance with Equation (1).

This is the background knowledge on which the radioheliograph was designed. Our objective was to study the outer corona and so we chose a frequency as low as possible subject to limitations imposed by ionospheric refraction. The frequency was thus set at $80 \mathrm{MHz}$ and other parameters as below:

Frequency: $80 \mathrm{MHz}$.

Diameter of telescope aperture: $3 \mathrm{~km}(=800$ wavelengths - sufficient to obtain a reasonable scanning beam size of $\left.3^{\prime} .9\right)$.

Field of view: $2^{\circ}$ diameter $(=4$ solar radii).

Polarization: both left and right circular polarization (required to study the magnetic properties).

Picture rate: 1 picture per second, (actually 1 pair of pictures per second, one in each polarization).

Daily coverage: Noon $\pm 2 \frac{1}{2}$ hours, a five hour observational day.

These requirements led to a rather novel design which, through its circular symmetry, seemed to be a very compact and quite elegant solution of the problem of 


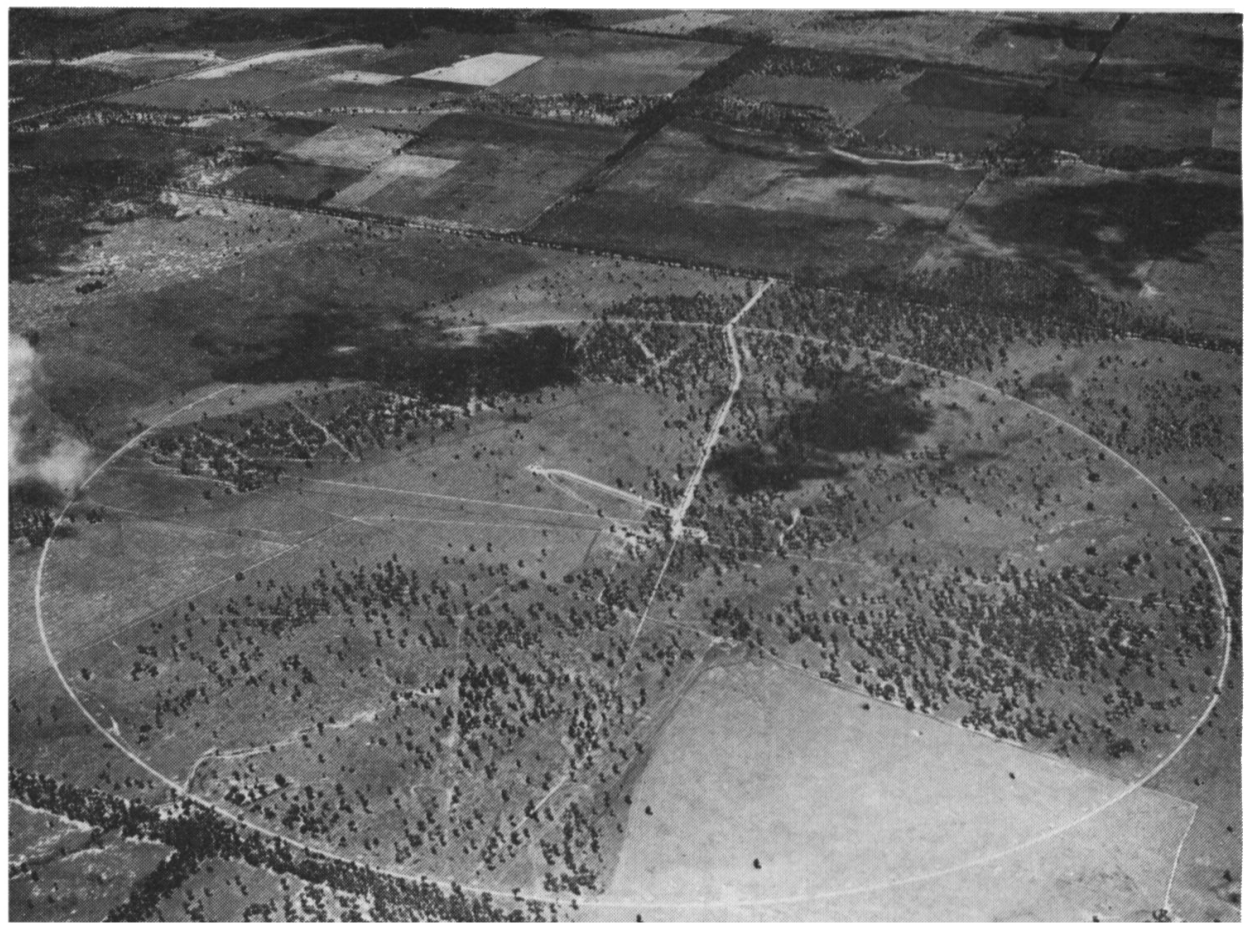

Fig. 3. Aerial photograph of the Culgoora radioheliograph.

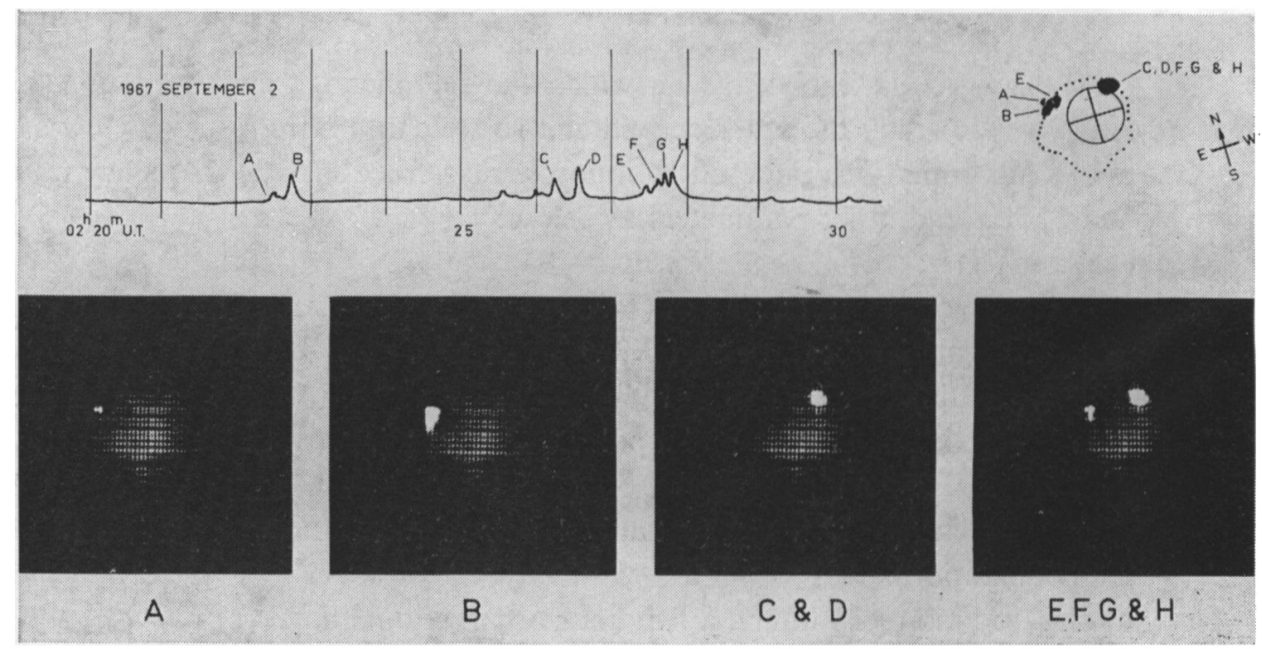

Fig. 4. $80 \mathrm{MHz}$ radioheliograms of a group of weak bursts. Note that the bursts originated in two widely separated, though apparently connected, sources. (Morimoto et al., 1967.) 
obtaining so large an aperture. It consists of ninety-six dishes, each of them $13 \mathrm{~m}$ in diameter, arranged around a circle of diameter $3 \mathrm{~km}$ (Figure 3); the whole thing acts as though it is one huge dish $3 \mathrm{~km}$ in diameter. This instrument taught us all about Bessel functions, and we offer it in years to come as a humble monument to nineteenth century mathematics. The instrument took five years to build, between the years 1962 and 1967. Since then we have continually upgraded it and nowadays it operates on three frequencies; 43,80 , and $160 \mathrm{MHz}$.

First I would like to show you results obtained during the very first hours of operation in September 1967. There was actually no appreciable solar activity in progress but on the flux record at one period some tiny little bursts $(\mathrm{A}, \mathrm{B}, \mathrm{C}, \ldots, \mathbf{H})$ appeared in isolation as shown on the record reproduced in the top left of Figure 4; it was the sort of insignificant record that was in the normal way destined for the waste-paper basket. What happened was quite extraordinary. As seen in the figure, bursts A, B and E appeared from one centre outside the optical limb, while bursts $C, D, F, G$ and $H$ appeared from a second, well separated centre. We were quite staggered at this extraordinary phenomenon of two distant, but apparently connected centres of activity. Surely it could not have been a coincidence. And, as it turned out, it was an indication of the shape of things to come.

\section{Observations of the Coronal Magnetic Field}

Now I would like to describe in a more or less systematic way some of the more interesting results which the instrument has revealed and, where possible, I will relate these coronal observations to what is seen optically in the photosphere and the chromosphere. It turns out not surprisingly, that one of the most important links between the photosphere and corona is the magnetic field which joins them.

So let us take a look at a sunspot group and its overlying region. In Figure 5 we see, at the top, an optical sunspot group, thein the $160 \mathrm{MHz}$ radiation of the associated bi-polar radio source and at the bottom the $80 \mathrm{MHz}$ source. In this figure the thin dotted contours refer to photospheric fields and the heavy contours to radio emission. You see how separated are the bi-polar radio components, and how at $80 \mathrm{MHz}$ they are a lot further apart than at $160 \mathrm{MHz}$ : at $80 \mathrm{MHz}$ there is a looser connection between the radio emission and the optical emission. The upper diagram of Figure 6 gives a three-dimensional model which directly interprets Figure 5 on the assumption that the emission is plasma radiation. Here we see the sunspot group and the loop fields drawn in such a way as to be consistent with the two polarized sources of radio emission. In fact it often happens that storms like this occur in very widespread parts of the Sun and, as it were, communicate with one another. It is quite clear that different centres of activity are connected by magnetic field lines and that storm centres become activated when electrons flow from one active centre to another. Occasionally one can see this in a very striking form with a transient burst of electrons which shows up first in one centre of activity and then in another. Indeed the observation shown in Figure 4 is the prototype example of this phenomenon. 
$N_{p} 1972$ October 30

\section{Circular RH ---- polarization $\mathrm{LH}$}
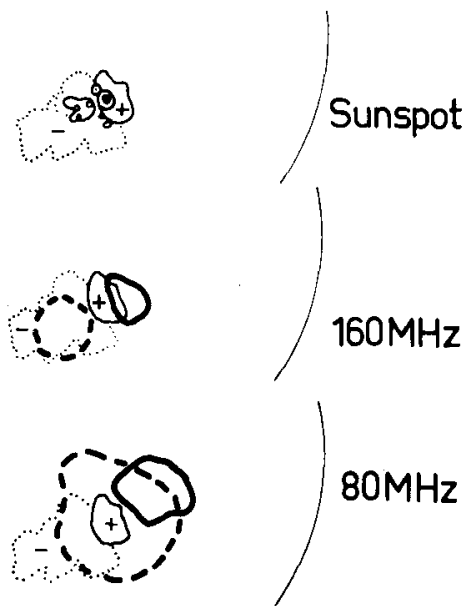

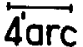

Fig. 5. A sunspot group observed in white light, $160 \mathrm{MHz}$ and $80 \mathrm{MHz}$ radiation. The outer (dotted) contour of the photospheric field is repeated in the other diagrams to provide positional reference.

(Kai, 1974.)
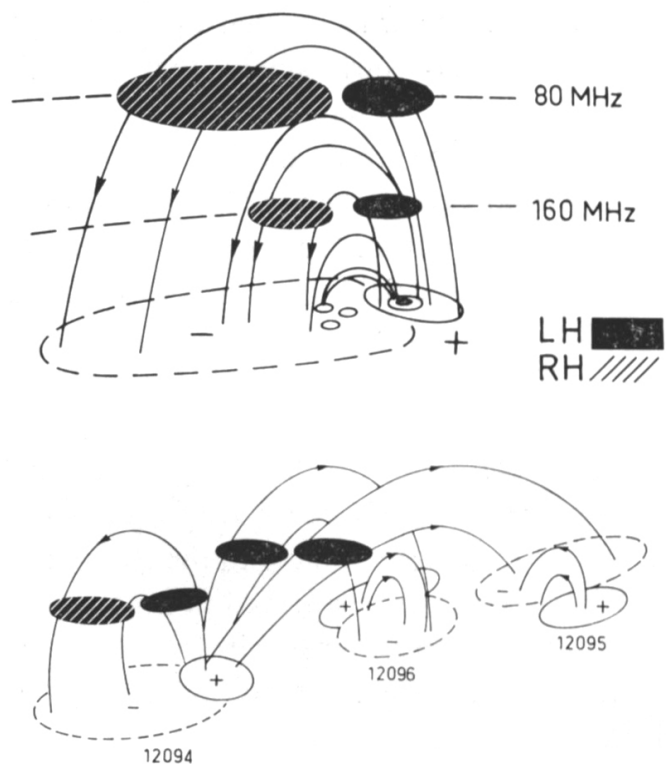

Fig. 6. Upper: Magnetic field configuration required to interpret records of the kind shown in Figure 5. The dark disks show the location of radio storm centres. Lower: Storm centres arising from magnetic fields that link distant centres of activity. 
Now occasionally we get a more dramatic situation in which we can actually 'see' a magnetic arch through its radio emission, and see it erupt and expand. Figure 7 is an example of such an expanding arch, following a flare, recorded at $80 \mathrm{MHz}$. The four pictures of the sequence are taken at intervals of about 4 or 5 minutes. We believe the arch is made radio emitting by virtue of supra-thermal and relativistic electrons trapped within it. As the expansion proceeds the arch is seen as three discrete sources: one unpolarized source at the top due to synchrotron radiation from relativistic electrons, and a pair of oppositely polarized sources near the feet of the arch attributed to plasma radiation excited by the supra-thermal electrons (cf. Figure 1).
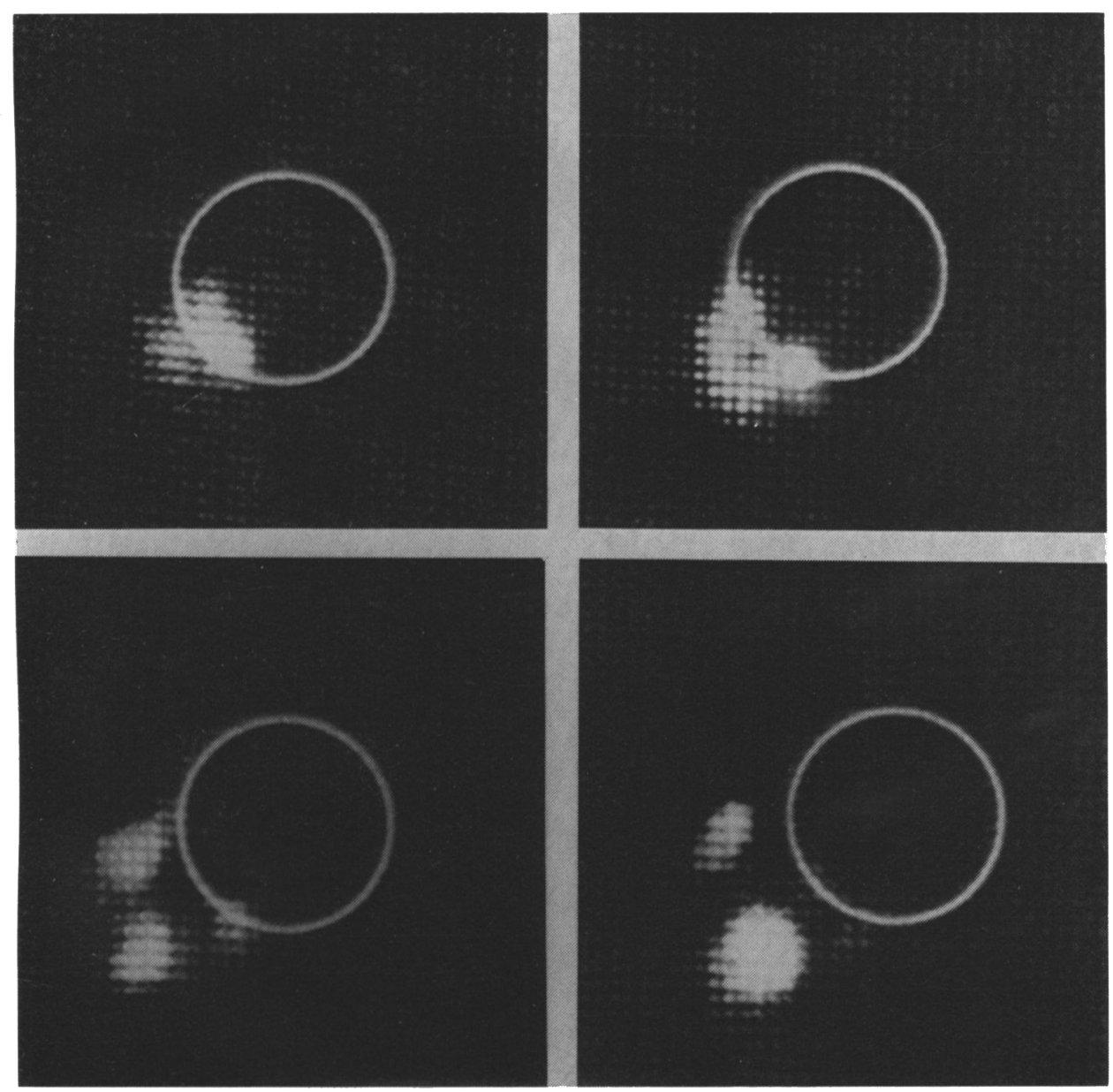

Fig. 7. A sequence $\left(\begin{array}{ll}1 & 2 \\ 3 & 4\end{array}\right)$ of $80 \mathrm{MHz}$ heliograms showing an activated magnetic arch which erupts and expands. The circles indicate the optical disk. The pictures are taken at times separated typically by $5 \mathrm{~min}$. (Wild, 1969.) 


\section{The Flash Phase of Solar Flares}

What I have just been talking about sets the scene, the magnetic environment, in which our other phenomena perform, and perhaps now we should turn to the central problem of the solar flare. I think one of the main contributions of radio observations has been to recognize two distinct phases of the flare and to give a physical description of each of these phases. The first phase of a solar flare, generally called the flash phase, is epitomized by the occurrence of a so-called type III burst (Figure 8), in which high frequencies are emitted slightly before low frequencies. It is characteristic that the spectrum may show two frequency bands corresponding to a fundamental frequency and its second harmonic; this indicates that we are observing plasma radiation (see Figure 1). Then, because there is a frequency drift from high to low frequencies, we must be dealing with electrons which are moving out from the Sun,

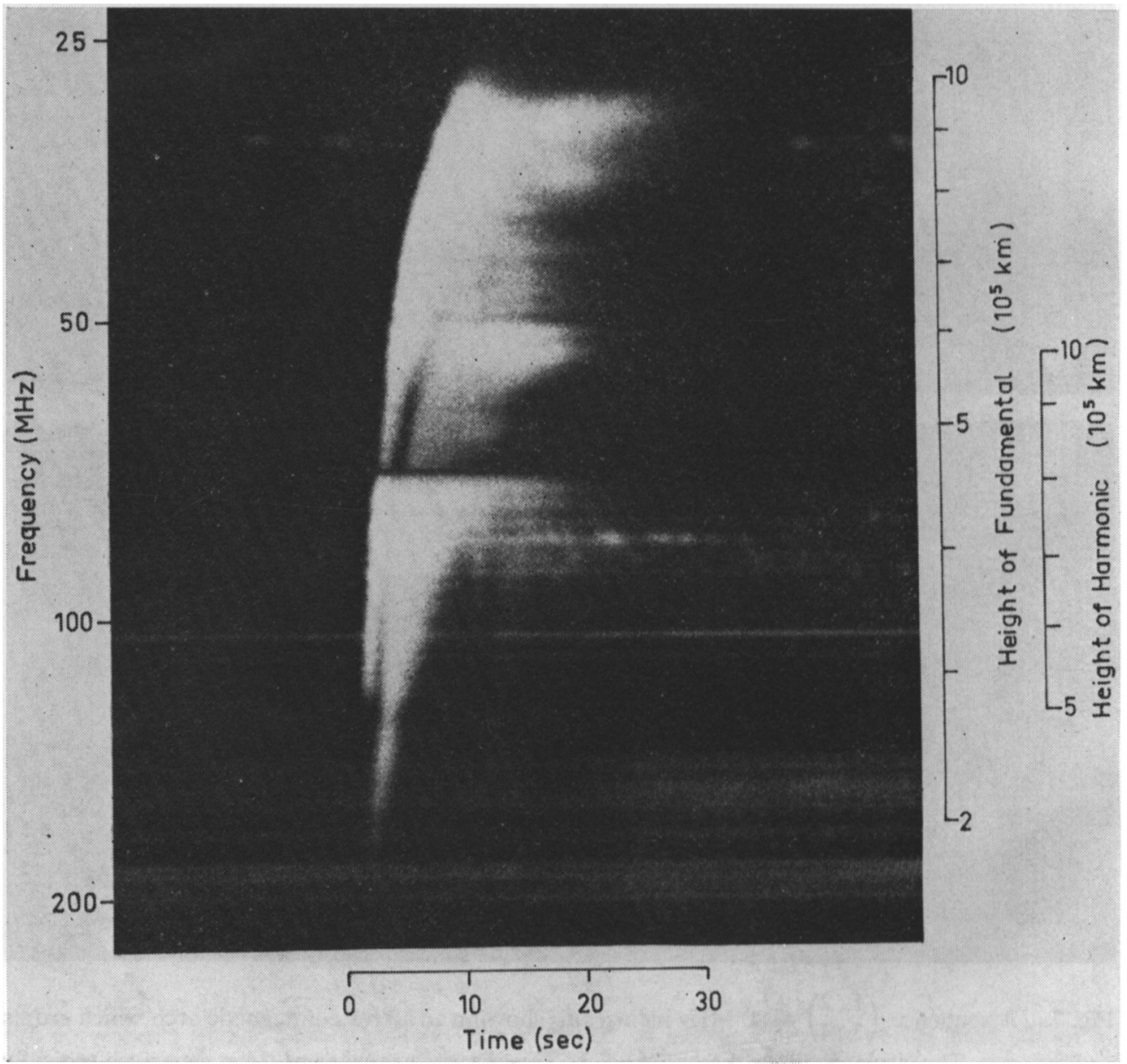

Fig. 8. Dynamic spectrum of a type III burst. 
from low down in the solar atmosphere to high up (see Figure 2). And when we use this frequency drift to calculate the velocity of the electrons, we find a value of about one-third the velocity of light. In other words we are dealing with a burst of electrons in the range $10-100 \mathrm{keV}$. The ejection of such a burst of electron lies at the heart of the flash phase which occurs at the very start of a solar flare.

To explain one way in which a type III burst can be generated, let me now develop the previous picture of the magnetic arch, by observing that when the arch extends high enough into the corona the solar wind comes along and grabs hold of the field lines and drags them out into a helmet structure, as shown in Figure 9. We believe a type III electron burst originates in a region where fields of opposite polarity come very close together; here some kind of magneto-hydrodynamics instability is responsible for the ejection of the burst of electrons which escape out along the neutral plane of the helmet. One contribution by the radioheliograph to our understanding of these bursts is direct evidence that the sources are often located in the neutral plane between opposing magnetic fields.

More recently the type III electron bursts have been picked up directly by satellites in interplanetary space or close to the Earth. And appropriately enough, as one would predict, there is a time delay of about half an hour between their detection on the Sun and at the Earth. The satellite observations have been studied especially by Bob Linn and his colleagues at the Space Science Laboratories at Berkeley. Now it is very

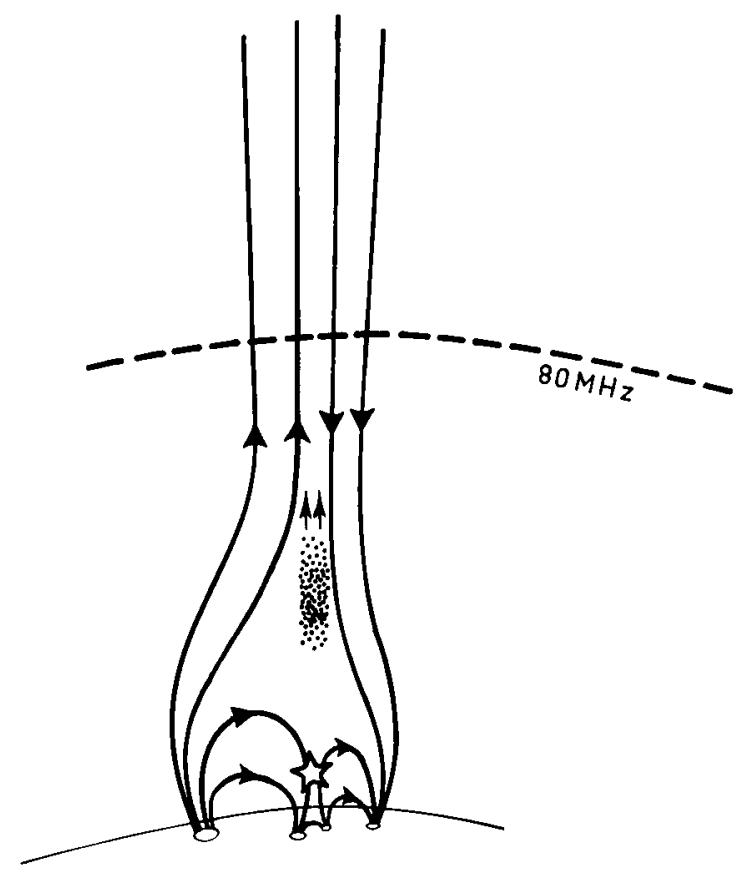

Fig. 9. Helmet structured magnetic field suited to the generation of electron pulses (cluster of dots) which generate type III bursts. The electrons may be generated by instabilities located where opposing lines of force are in close proximity (e.g. at star). 
interesting to see the radioheliograph pictures of the type III bursts that correspond to detectable electron bursts. Some are shown in Figure 10 where one is actually seeing the pulses of electrons as they move out through the solar corona. You notice the remarkable fact that every one of them is on the western limb of the Sun. This makes very good sense when one takes into consideration the Archimedes spiral form of the interplanetary field (Figure 11): for particles to arrive near the Earth they have to be ejected from the west limb. Nowadays, type III bursts are studied very effectively by means of very low frequency observations taken from satellites which can extend the range of heights right through interplanetary space. And through some quite brilliant observations by Joe Fainberg, Bob Stone and others at NASA's Goddard Space Flight Centre, as well as at the University of Michigan, one can trace the electron trajectories right along the Archimedes spiral to the Earth's orbit.

That is all I am going to say about the flash except to point out that the bursts of electrons that we pick up not only cause type III bursts and produce detectable electrons in space, but similar electrons traveling inwards towards the photosphere produce microwave bursts and $\mathrm{X}$-rays which in turn cause the sudden ionospheric disturbance effects on the Earth. And so a whole range of phenomena can be explained simply from the one basic cause.

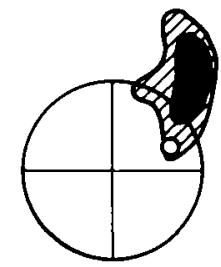

1969 FEB. 26

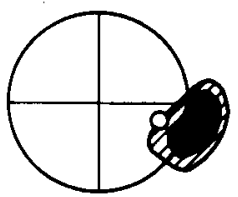

1970 MAR. 12

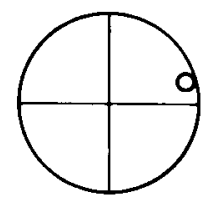

$1970_{2}$ SEPT. 10

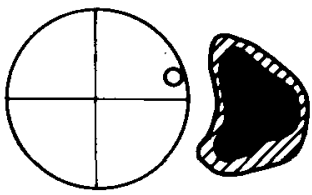

1969 MAY 29

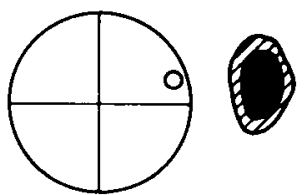

1970 JULY 29

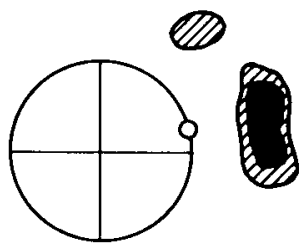

1970 SEPT. 11

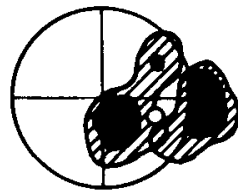

1969 ОСТ. 13
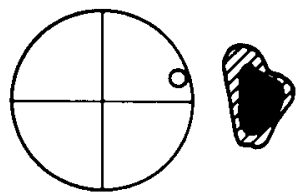

1970 SEPT. 10
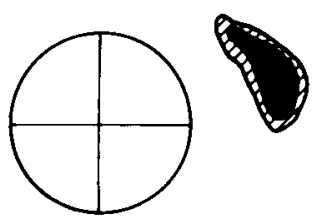

1970 SEPT. 13

Fig. 10. $80 \mathrm{MHz}$ heliograms of nine type III bursts which accompanied electrons detected directly from satellites. (After I. D. Palmer and R. P. Lin, to be published). 


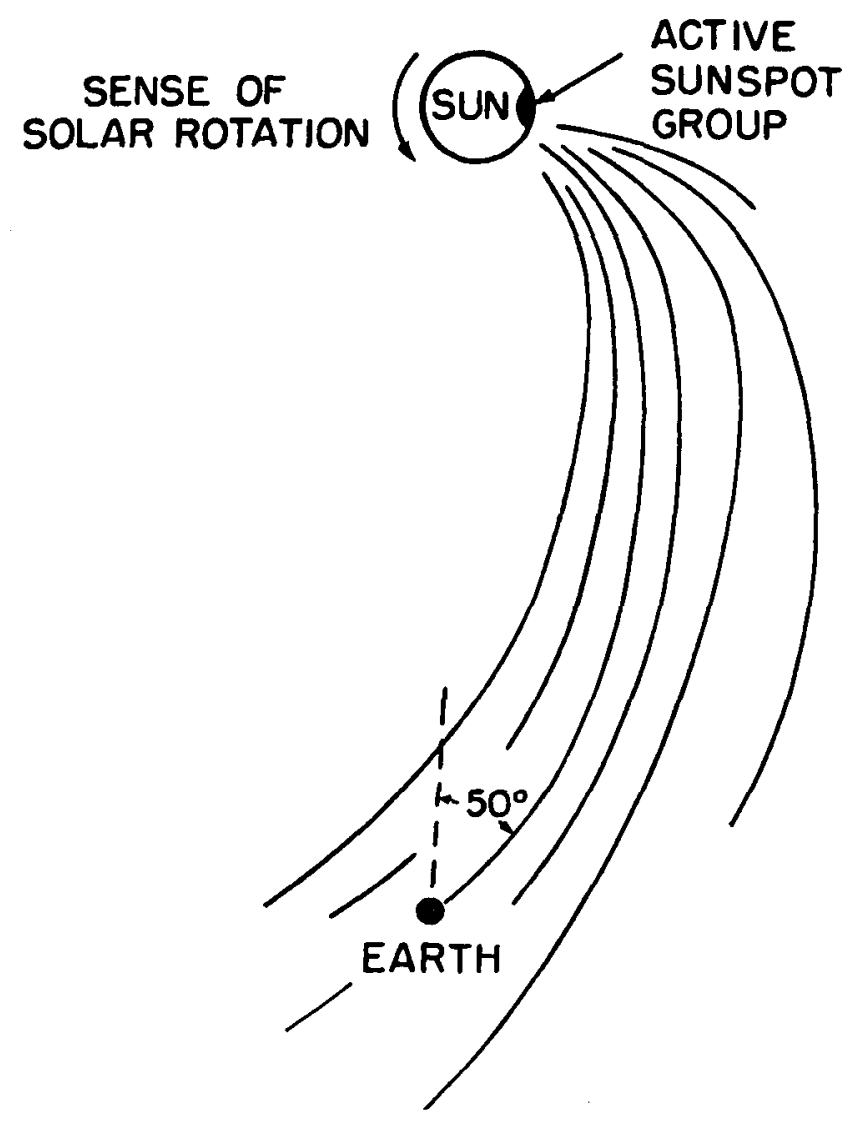

Fig. 11. Archimedes spiral traced out by the interplanetary magnetic field.

\section{The Blast Wave}

We now pass onto the second phase of a solar flare. At the same time as the explosive instability causing the type III burst takes place, a blast wave moves out at a speed of the order of a $100 \mathrm{~km} \mathrm{~s}^{-1}$ and forms into a sharply defined shock wave. As it advances we can see on the radio spectrum the spectacle of a type II solar burst, a quite beautiful

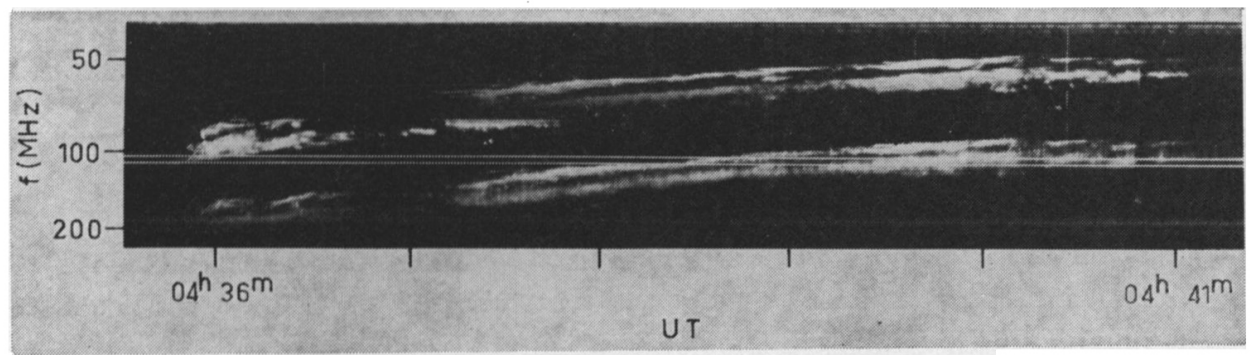

Fig."12. Dynamic spectrum of a type II burst. 


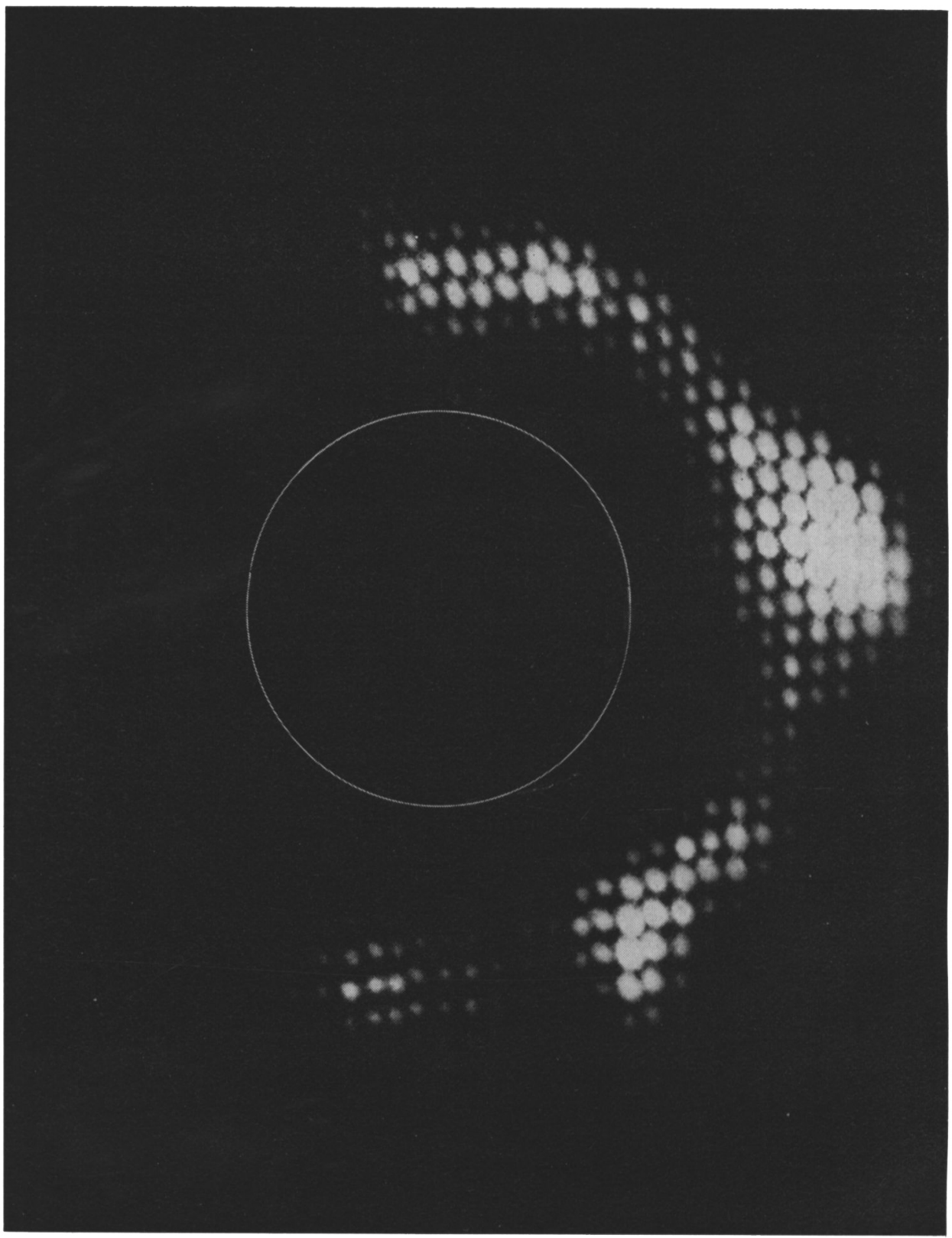

Fig. 13. $80 \mathrm{MHz}$ heliogram taken during the early stage of a great outburst on 30th. March 1969 consisting mainly of type II bursts. The flare occurred beyond the west limb. (Smerd, 1970.)

phenomenon. An example is shown in Figure 12 and we note once again the presence of fundamental and harmonic bands and of frequency drift just as with the type III burst, but now with a different time scale about 200 times slower. This difference corresponds to that between velocities of $1000 \mathrm{~km} \mathrm{~s}^{-1}$ and one-third the velocity of 
light. This is really the definitive phenomenon of the second phase. When the shock front reaches the $80 \mathrm{MHz}$ level we can expect it to register on our radioheliograph. Figure 13 is a dramatic example of a radioheliogram of an outburst in which two type II bursts occurred. The shock waves travelled out from the source near the limb - actually behind the limb in this case; the event, recorded on 1969 March 30, was accompanied by intense proton emission.

With radio observations we can detect the shock wave from a flare by the type II burst quite as a matter of routine. It is only rather rarely that one can pick up the shock wave by optical means; some fine examples have been recorded at the Lockheed Observatory by Moreton and Ramsey and others, and a clear association with type II bursts has been established.

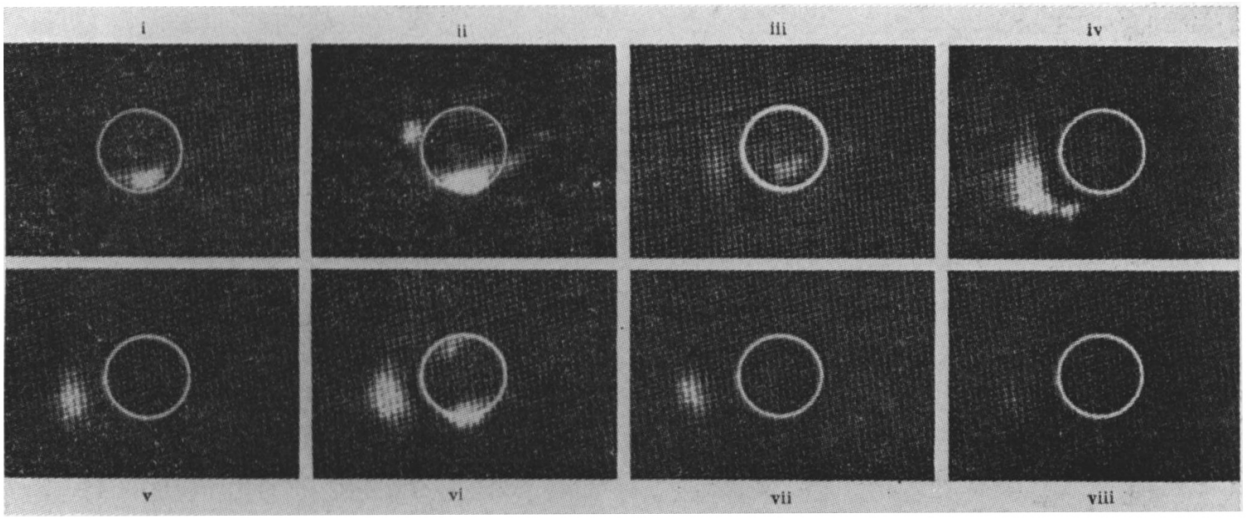

Fig. 14. Sequence of $80 \mathrm{MHz}$ heliograms showing a type II burst (i-iii) followed by a synchrotron burst above an eruptive prominence (iii-viii) believed to be triggered by the same shock wave.

(Wild et al., 1968.)

It is clear from optical records, that a flare can make its presence known across large regions of the solar sphere through the propagation of the shock wave. Radio observations go one step further and show that shock waves can actually trigger the release of high-energy particles at very great distances. A classic example at $80 \mathrm{MHz}$ is shown in the sequence of pictures in Figure 14: type II radio emission first occurs above a flare (located on the 5 o'clock vector, regarding the solar disc as a clock face). Minutes later an optical prominence erupts on the limb (on the 8 o'clock vector) and this in turn is accompanied by $800 \mathrm{MHz}$ synchrotron radiation from a source high above the eruptive prominence. The whole sequence can be explained in terms of the effects of a shock wave emanating from the original flare. Figure 15 illustrates how coronal shock waves may trigger such prominence eruptions and, indeed, other flares. Finally I should not forget to mention that one of the most distant interactions involving the shock wave is when it strikes the Earth's atmosphere and produces aurorae and magnetic storms on the Earth. 


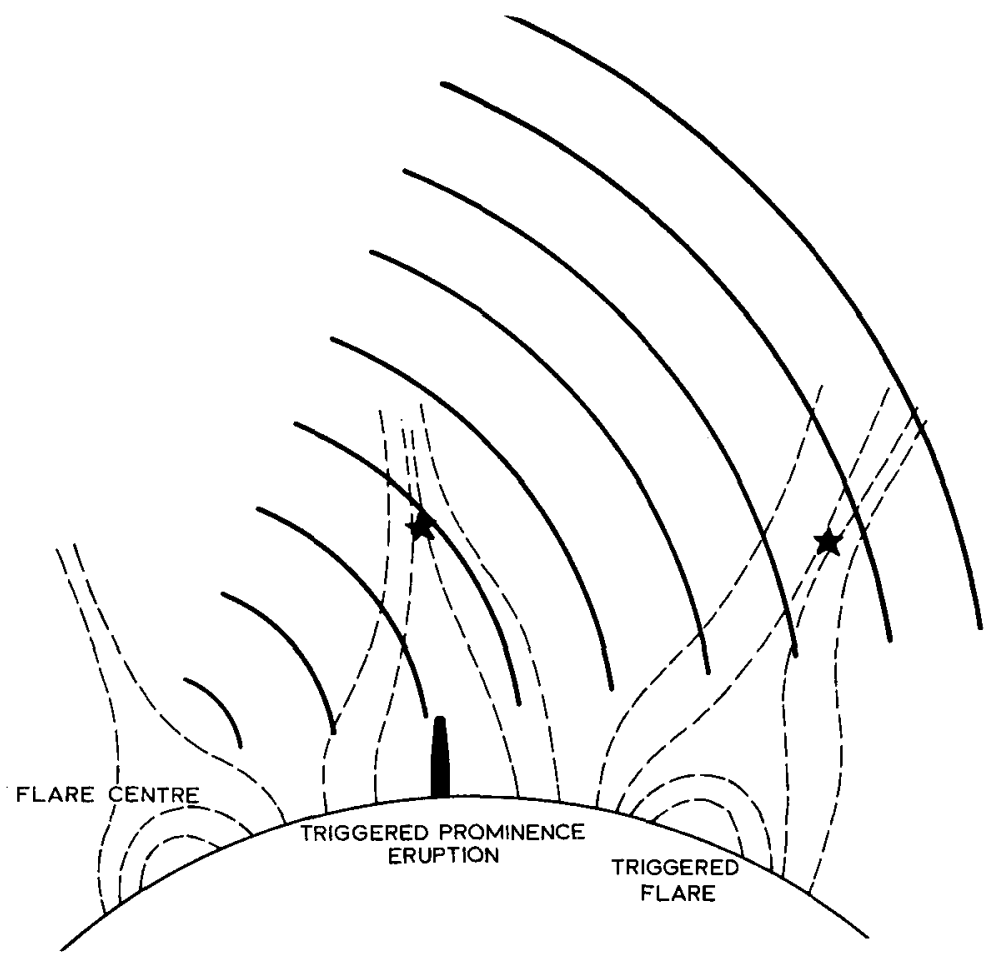

Fig. 15. Diagram showing how advancing shock waves may trigger instabilities (stars) to cause prominence eruptions and other flares.

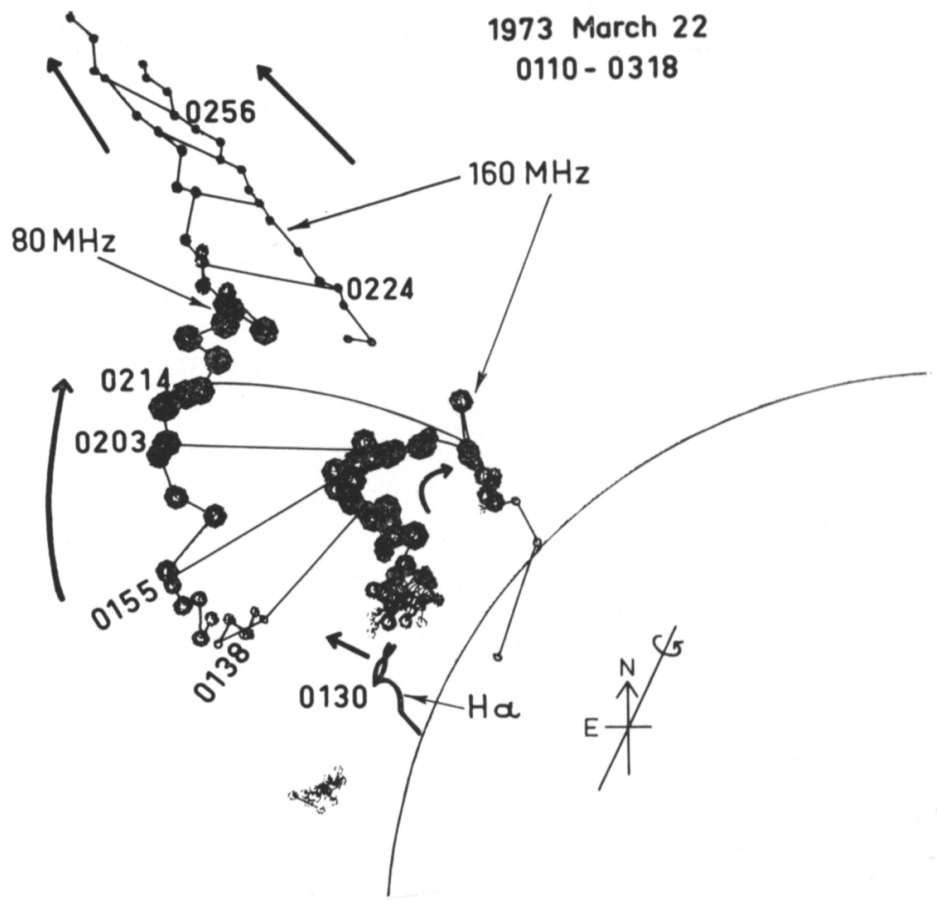

Fig. 16. Diagram showing the evolution of an event in which a slow $\left(100 \mathrm{~km} \mathrm{~s}^{-1}\right)$ disturbance prompted the start of a radially ejected isolated source (plasmoid?). (McLean, 1974.) 


\section{A Third Phase to the Solar Flare?}

Up till very recently it was widely believed that almost all the important phenomena caused by flares could be traced to the two phases we have just discussed. On the other hand, from time to time one sees peculiar blobs of radiating matter leave the Sun not at supersonic speeds but at rather moderate speeds, a mere $250 \mathrm{~km} \mathrm{~s}^{-1}$, which is about the Alfvén velocity in the corona. And also one sees optical phenomena such as flare surges which have velocities, typically of the order of $100 \mathrm{~km} \mathrm{~s}^{-1}$, much slower than coronal shock waves. Now I believe that recent radioheliograph results have begun to make it clear that we should perhaps start thinking of a third basic phase of the flare associated with a slower disturbance. Figure 16 shows what might turn out to be a rather definitive record that depicts a distinctly slower type of disturbance quite different from the disturbances we have previously discussed. Following an $\mathrm{H} \alpha$ surge (shown at $01^{\mathrm{h}} 30^{\mathrm{m}}$ ) with velocity about $60-100 \mathrm{~km} \mathrm{~s}^{-1}$, the radio observations at 160 and $80 \mathrm{MHz}$ reveal what might be interpreted as a column of gas

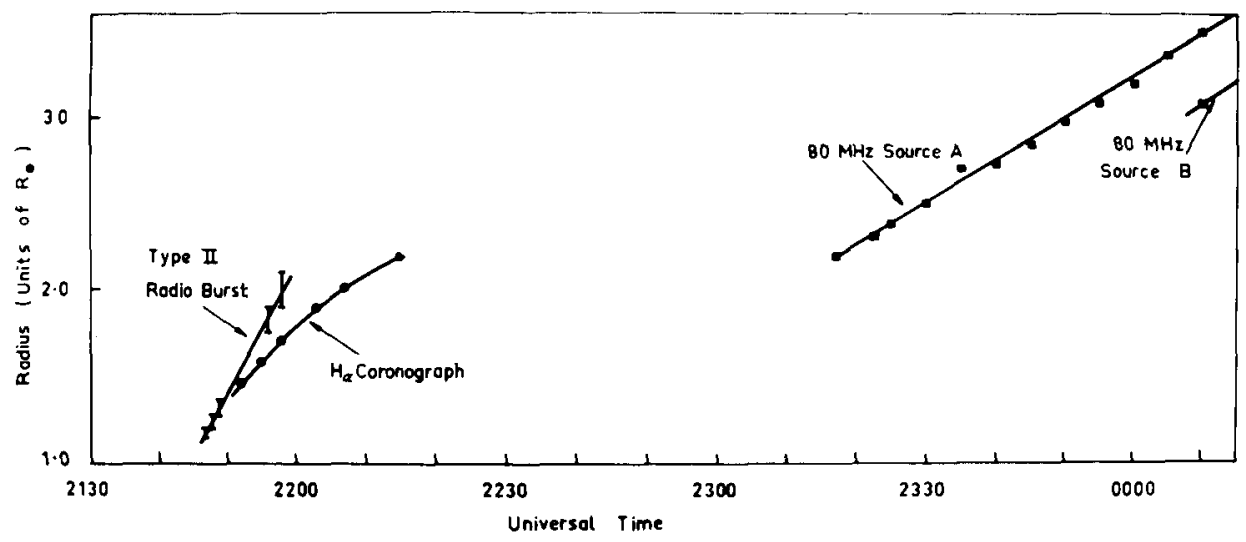

Fig. 17. Height/time analysis of the event of 1-2 March 1969 ('Westward Ho'), showing the 1 hour's time delay between type II shock wave and later ejection. (Riddle, 1970.)

beginning to move out at $01^{\mathrm{h}} 38^{\mathrm{m}}$. The column is channelled round an arc and excites the 160 and the $80 \mathrm{MHz}$ radiation at two different levels, presumably corresponding to the plasma levels. The speed of travel is still about $100 \mathrm{~km} \mathrm{~s}^{-1}$ (very slow) until it reaches what looks like the top of an arch structure. Perhaps you can imagine a helmet structure being drawn around this configuration - it is certainly very suggestive of it. When the disturbance reaches the top of the arch, something else happens; a discrete disturbance, bipolar in structure, moves out radially at about $250 \mathrm{~km} \mathrm{~s}^{-1}$. Now in the past few years we have witnessed a number of such radially moving disturbances, sometimes reaching solar distances of as much as $5-6 R_{\odot}$. We think perhaps these ejections are self-contained plasmoids but it has always been something 

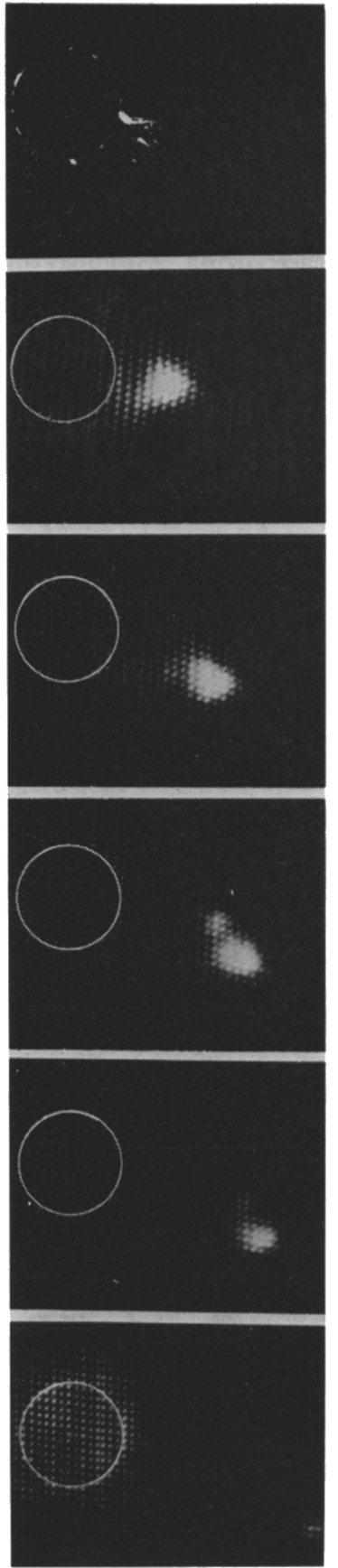

Fig. 18. Sequence of $80 \mathrm{MHz}$ heliograms during the event summarized in Figure 17. The top picture is the associated $H \alpha$ prominance (flare spray) recorded at the University of Hawaii. 
of a puzzle that although the ejections come from the general direction of the flare centre the timing is often quite wrong for the ejection to have come from the initial flare explosion. Typically, as illustrated by Figure 17 (which depicts the rather wellknown event nicknamed 'Westward Ho'), there is a one-hour delay between the origin of the shock wave and the origin of the slow 'third phase' ejection. The phenomenon in Figure 16 now offers an explanation of the missing hour. It suggests that the plasmoid (if such be the disturbance) is ejected not from the flare centre but rather from an instability located at the point in the helmet structure where the top of the arch meets the base of the radial streamer. And that the instability is triggered by a slow $\left(100 \mathrm{~km} \mathrm{~s}^{-1}\right)$ column of gas which is first manifest optically as a flare surge and is later seen in radio emission as a source climbing up and around the magnetic arch.

To end this discourse let us take a look at the movie of Westward Ho! itself (Figure 18) travelling out and out at $270 \mathrm{~km} \mathrm{~s}^{-1}$ through more than 2 million kilometres. The accompanying music clearly ought to be the William Tell overture.

Mr Chairman, I think I should finish at this stage and leave you in a position half way between the Sun and the Earth. And although I have taken you through quite a long road of comples and varied phenomena, I hope you will grant that the main contribution of this work is basically one of unification and simplification.

\section{References}

Kai, K. and Sheridan, K. V.: 1974, Solar Phys., in press.

McLean, D. J.: 1973, Proc. Astron. Soc. Australia 2, 222.

Morimoto, M., Sheridan, K. V., and Wild, J. P.: 1967, Proc. IEEE Australia 28, No. 9.

Riddle, A. C.: 1970, Solar Phys. 13, 448.

Smerd, S. F.: 1970, Proc. Astron. Soc. Australia 1, 305.

Wild, J. P. (ed.): 1967, Proc. IEEE Australia 28, No. 9.

Wild, J. P.: 1969, Solar Phys. 9, 260.

Wild, J. P.: 1970, Proc. Astron. Soc. Australia 1, 365.

Wild, J. P. and Smerd, S. F.: 1972, Ann. Rev. Astron. Astrophys. 10, 159.

Wild, J. P., Sheridan, K. V., and Kai, K.: 1968, Nature 218, 536. 